\title{
Feasibility and Acceptability of a Home-based Resistance Training Intervention in Adolescent and Young Adult Hematopoietic Cell Transplant Survivors
}

\author{
Tyler Ketterl ${ }^{1}$, Sheri Ballard ${ }^{2}$, Miranda Bradford $^{3}$, Eric Chow ${ }^{2}$, Kari Jenssen ${ }^{2}$, Sam \\ Myers $^{2}$, Abby Rosenberg${ }^{1}$, Matt Van Doren ${ }^{2}$, and K. Scott Baker ${ }^{4}$ \\ ${ }^{1}$ Seattle Children's Hospital \\ ${ }^{2}$ Fred Hutchinson Cancer Research Center \\ ${ }^{3}$ Seattle Children's Research Institute \\ ${ }^{4}$ University of Washington School of Medicine
}

February 2, 2021

\begin{abstract}
BACKGROUND Adolescent and young adult (AYA) hematopoietic cell transplantation (HCT) survivors are at increased risk of metabolic syndrome and lean body mass (LBM) deficits. Resistance training (RT) is a potential intervention to improve LBM, metabolic fitness and reduce risk of cardiovascular disease. PROCEDURE Eligible participants ages 13-39 years, 80-120 days post-HCT, transfusion independent, and prednisone dose $<1 \mathrm{mg} / \mathrm{kg} /$ day were approached. Baseline assessments of body composition (DXA), anthropometrics and strength testing were completed and participants were taught a 12-week, homebased RT intervention with weekly remote coaching. Follow-up assessments were at day +200 (FU1) and +365 post-HCT (FU2). Feasibility targets were 1) 60\% enrollment of approached patients, 2) $80 \%$ completion of weekly phone calls and 3 ) $80 \%$ completion of the RT intervention and FU1 assessments. Acceptability was measured by recommendation of the intervention to an AYA HCT survivor. RESULTS Twenty of 31 (65\%) eligible AYAs enrolled. Two participants failed to complete baseline measurements ( 1 =scheduling barriers, $1=$ passive refusal) and 4 participants who completed baseline assessments did not receive the intervention $(2=$ medical reasons, $2=$ no longer interested). Of the 13 who received the intervention, 11 ( $85 \%)$ completed FU1 and completed $88.5 \%$ of coaching calls. LBM $(\mathrm{kg})$ increased or remained unchanged in $9 / 9$ participants with complete body composition data at FU1 (mean $1.1 \mathrm{~kg} ; 95 \% \mathrm{CI}$ : 0.4,1.9). All participants who completed FU1 reported they would recommend the intervention to an AYA HCT survivor. CONCLUSIONS A home-based RT intervention in AYA HCT survivors early post HCT is both feasible and acceptable and may maintain or increase LBM.
\end{abstract}

\section{TITLE :}

Feasibility and Acceptability of a Home-based Resistance Training Intervention in Adolescent and Young Adult Hematopoietic Cell Transplant Survivors

\section{Authors}

Tyler G. Ketterl, MD, MS $1,2,3$

Sheri Ballard, $\mathrm{MPH}^{3}$

Miranda C. Bradford, MS 4

Eric J. Chow, MD, MPH ${ }^{1,2,3}$

Kari Jenssen ${ }^{3}$ 
Sam Myers, MS ${ }^{5}$

Abby R. Rosenberg, MD, MS, MA ${ }^{1,2,3}$

Matt Van Doren ${ }^{5}$

K. Scott Baker, MD, MS ${ }^{1,2,3}$

1. Cancer and Blood Disorders Center, Seattle Children's Hospital, Seattle, WA

2. Division of Pediatric Hematology/Oncology, Department of Pediatrics, University of Washington, Seattle, WA

3. Clinical Research Division, Fred Hutchinson Cancer Research Center, Seattle, WA

4. Core for Biostatistics Epidemiology and Analytics in Research, Seattle Children's Research Institute, Seattle, WA

5. Exercise Research Center, Fred Hutchinson Cancer Research Center, Seattle, WA

\section{Corresponding Author:}

Tyler G. Ketterl, MD, MS

Seattle Children's Hospital

MB.8.501 PO Box 5371,

Seattle, WA 98145-5005 Phone: (206) 987-2146

Fax: 206-987-3946

Email: Tyler.Ketterl@SeattleChildrens.org Abstract: 249 words Main Text: 2620 words 4 Tables

2 Figures

Running Head :

Home-based resistance training in AYA HCT survivors

Keywords:

Adolescent and Young Adult, Resistance Training

Abbreviations Table

\begin{tabular}{ll}
\hline AYA & adolescent and young adult \\
\hline CVD & cardiovascular disease \\
DXA & dual X-ray absorptiometry \\
FHCRC & Fred Hutchinson Cancer Research Center \\
FU1 & follow-up 1 \\
FU2 & follow-up 2 \\
GVHD & graft versus host disease \\
HCT & hematopoietic cell transplantation \\
LBM & lean body mass \\
PFM & percent fat mass \\
RT & resistance training \\
TBI & total body irradiation \\
\hline
\end{tabular}

\section{ABSTRACT}

BACKGROUND

Adolescent and young adult (AYA) hematopoietic cell transplantation (HCT) survivors are at increased 
risk of metabolic syndrome and lean body mass (LBM) deficits. Resistance training (RT) is a potential intervention to improve LBM, metabolic fitness and reduce risk of cardiovascular disease.

\section{PROCEDURE}

Eligible participants ages 13-39 years, 80-120 days post-HCT, transfusion independent, and prednisone dose $<1 \mathrm{mg} / \mathrm{kg} /$ day were approached. Baseline assessments of body composition (DXA), anthropometrics and strength testing were completed and participants were taught a 12-week, home-based RT intervention with weekly remote coaching. Follow-up assessments were at day +200 (FU1) and +365 post-HCT (FU2). Feasibility targets were 1) $60 \%$ enrollment of approached patients, 2) $80 \%$ completion of weekly phone calls and 3) $80 \%$ completion of the RT intervention and FU1 assessments. Acceptability was measured by recommendation of the intervention to an AYA HCT survivor.

\section{RESULTS}

Twenty of 31 (65\%) eligible AYAs enrolled. Two participants failed to complete baseline measurements ( $1=$ scheduling barriers, $1=$ passive refusal) and 4 participants who completed baseline assessments did not receive the intervention $(2=$ medical reasons, $2=$ no longer interested $)$. Of the 13 who received the intervention, $11(85 \%)$ completed FU1 and completed $88.5 \%$ of coaching calls. LBM $(\mathrm{kg})$ increased or remained unchanged in 9/9 participants with complete body composition data at FU1 (mean $1.1 \mathrm{~kg}$; 95\%CI: 0.4,1.9). All participants who completed FU1 reported they would recommend the intervention to an AYA HCT survivor.

\section{CONCLUSIONS}

A home-based RT intervention in AYA HCT survivors early post HCT is both feasible and acceptable and may maintain or increase LBM.

\section{INTRODUCTION:}

There were more than 678,000 adolescent and young adult (AYA) cancer survivors in the US in 2019, and this number is projected to increase by more than $30 \%$ in the next 10 years. ${ }^{1}$ Unfortunately, one-third of childhood cancer survivors have severe or life-threatening medical complications 30 years after diagnosis and early mortality from cardiovascular disease (CVD) is one of the leading causes of non-relapse mortality. ${ }^{2-5}$ The growing number of survivors raises the importance of developing interventions to reduce these adverse late effects of cancer treatment, including after hematopoietic cell transplantation (HCT) ${ }^{1,6}$ HCT survivors are at increased risk of metabolic syndrome (central obesity, insulin resistance, glucose intolerance, dyslipidemia, and hypertension $),{ }^{7-10}$ lean muscle mass deficits and sarcopenic obesity compared to sibling controls. ${ }^{11,12}$ In a recent study, an exercise intervention including resistance training (RT) in adult breast cancer survivors was shown to attenuate metabolic syndrome, sarcopenic obesity, and circulating biomarkers. ${ }^{13}$ Lower muscle mass and higher central adiposity are highly predictive of insulin resistance and are potential targets for interventions designed to enhance metabolic fitness and reduce risk of CVD in adolescent cancer survivors.

Resistance training (RT) is a form of physical activity designed to improve muscular fitness by exercising a muscle or a muscle group against external resistance including traditional free weights and dumbbells, weight machines, body weight, elastic tubing, medicine balls, or even common household products like milk jugs filled with sand or soup cans. ${ }^{14}$ RT has been established as effective in increasing lean body mass, preventing abdominal and total fat gain, and reducing markers of inflammation and other cardio-metabolic risk factors in healthy adults. ${ }^{15-18}$ Studies in adults have demonstrated resistance training regimens are feasible and effective at improving abdominal adiposity, lipid metabolism and fat mass. ${ }^{15,19}$ Muscular strength has also been identified as an independent and powerful predictor of better insulin sensitivity in children. ${ }^{20}$

A 2019 systematic review of physical activity in AYA cancer patients highlighted the lack of high-quality studies aimed at improving physical functioning in this population, ${ }^{21}$ and even fewer studies evaluating RT interventions in these survivors. ${ }^{22,23}$ Furthermore, there are few studies evaluating RT interventions in pediatric and AYA HCT survivors and none of a home based intervention, which is likely to be more 
feasible, acceptable and generalizable than in-hospital supervised programs. ${ }^{22,23}$ Here in a small pilot study, we evaluate the feasibility and acceptability of a RT intervention uniquely suited to the needs of AYA cancer survivors, consisting of a brief in-person training at study entry followed by a home-based regimen for the duration of the study with remote coaching.

\section{METHODS}

\section{Study Design}

We conducted a single arm pilot study of an exercise intervention evaluating the feasibility and acceptability of home-based resistance training intervention in AYA HCT survivors. We recruited participants from the Seattle Children's Hospital, Seattle Cancer Care Alliance and Fred Hutchinson Cancer Research Center (FHCRC) in Seattle, WA between November 2018 and February 2020. We targeted a sample size of 2025 participants. The intervention period was 12 weeks. The primary endpoint was feasibility which was assessed through enrollment of approached and eligible patients, completion of weekly follow-up phone calls and completion of the full intervention among enrolled patients. As secondary endpoints, acceptability, change in body composition, and muscular strength were described.

\section{Eligibility}

Patients who were at least 80 days but less than 120 days out from HCT were recruited for participation in the study. Eligibility criteria included 13-39 years old, ambulatory (able to walk) and without medical contraindication to increasing physical activity, transfusion independent, and $<1 \mathrm{mg} / \mathrm{kg} /$ day of prednisone and on a tapering schedule. After providing written informed consent (and assent if $<18$ years of age), patients were enrolled on the study. The study was approved by the IRB at Fred Hutchinson Cancer Research Center (no. 10046) and conducted in accordance with the ethical standards of the Declaration of Helsinki. It is registered at ClinicalTrials.gov (NCT03672981).

\section{Intervention}

The RT exercise prescription was uniquely formulated for AYA patients according to RT principals outlined by the American College of Sports Medicine strength training guidelines. ${ }^{24}$ Participants were offered up to three initial in-person exercise teaching sessions with an exercise physiologist at the FHCRC Exercise Research Center where they were taught the home-based exercise program. The RT intervention was tailored to the participant's baseline strength assessments by the exercise physiologist. Each participant was prescribed a progressive resistance program, with participants completing 1-2 sets of 8 to 10 exercises, 8 to 12 repetitions of each exercise, 2-3 days per week. Participants were given graded resistance bands to complement body weight exercises to target all major muscle groups with the primary goal of increasing muscle mass. An exercise physiologist then conducted weekly follow-up phone calls with each participant to assess adherence to the exercise program and to provide support including identifying and overcoming barriers to exercise, goal setting, assess for adverse events, and providing motivation to exercise.

\section{Outcome Measures}

Baseline assessments were performed within 2 weeks of study enrollment. The primary outcomes were assessed at Follow-up 1 (FU1) within 2 weeks after the 12-week intervention period. Outcomes were also assessed secondarily at Follow-up 2 (FU2) within 4 weeks of day +365 post HCT. At all timepoints, anthropometrics, body composition, and strength testing were measured. For assessment of feasibility, we traced the number of eligible participants who enrolled in the study, the completion of weekly follow-up phone calls with participants and the number of participants who completed the 12-week intervention and FU1. To assess acceptability, one-on-one, semi-structured interviews were performed by a clinical research assistant via telephone following completion of the 12-week RT intervention. The interviews were conducted following a standardized script. Sessions were digitally recorded, transcribed verbatim and analyzed. Participants were asked "Would you recommend this program to other adolescent/young adult hematopoietic cell transplant cancer survivors?" Acceptability was measured by a response of "yes" or "I would" recommend the intervention to another AYA HCT survivor. 


\section{Body Composition}

Heigh and weight were obtained in the FHCRC Exercise Research Center or extracted from the medical record within 1 week of the evaluation time points. Dual x-ray absorptiometry (DXA) was used to evaluate body composition including lean body mass (LBM) and percent fat mass (PFM).

\section{Strength Testing}

Strength and endurance assessments were performed at the FHCRC Exercise Research Center under the direct supervision of an exercise physiologist. Assessments included hand grip strength, 30-second chair stand assessment, one repetition maximum chest press, one repetition maximum leg press and a 6-minute walk test.

\section{Primary Disease and Transplant Related Data}

Primary disease and transplant related data were extracted from the electronic medical record. Data on donor source, conditioning regimen, radiation exposure, and steroid exposure for treatment of graft versus host disease (GVHD) were also collected.

\section{Statistical analysis}

Participants' age, gender, and socio-demographic characteristics were summarized using descriptive statistics, as were participants' medical characteristics, including underlying diagnosis, HCT conditioning regimen and donor source. Feasibility was defined as: (1) at least $60 \%$ enrollment of approached and eligible patients; (2) at least $80 \%$ completion of the full intervention among enrolled patients; and (3) at least $80 \%$ completion of weekly follow-up phone calls. Absolute and percentage change for muscle strength and body composition outcomes were calculated for the periods baseline to FU1 and baseline to FU2. Changes were summarized using means and 95\% confidence intervals together with the number of participants experiencing no change, benefit or decline. Statistical analyses were performed using Stata version 16 (StataCorp., College Station, TX).

\section{RESULTS}

\section{Feasibility and Acceptability}

Between November 2018 and February 2020, 91 HCT survivors were screened for eligibility, 31 were eligible, 30 were approached, and 20 survivors $(64.6 \%)$ were enrolled in the study. Study enrollment was stopped in February 2020 due to the SARS-CoV-2 pandemic limiting enrollment of up to an additional 5 participants. Participants ranged in age from 16-39 years, $6(30 \%)$ were female, and $10(50 \%)$ had a 4-year college degree or higher. (Table 1) Ten participants $(50 \%)$ had a primary diagnosis of acute lymphoblastic leukemia, $5(25 \%)$ acute myeloid leukemia, $4(20 \%)$ Hodgkin lymphoma, and 1 participant had a germ cell tumor. Most participants $(\mathrm{n}=14,70 \%)$ received total body irradiation (TBI) and had an allogeneic donor source $(\mathrm{n}=16,80 \%)$. (Table 2) Seventeen participants (85\%) completed baseline measurements. Of the 3 who did not complete baseline measurements, 2 participants cited lack of evening/weekend FHCRC Exercise Research Center hours and 1 passively refused participation after enrollment. Of the participants who completed baseline assessments, $11(65 \%)$ completed FU1. Of the 6 participants who did not complete FU1 measurements, 2 had disease relapse, 1 suffered a non-intervention related medical condition preventing exercise, 2 declined further follow-up, and 1 declined to follow-up due to the SARS-CoV-2 pandemic. Six (55\%) of the participants who completed FU1 required steroids for GVHD during the intervention period. There were no significant adverse events during the study. Of the 11 participants who completed assessments at FU1, 10 completed assessments at FU2. The patient who did not complete FU2 had disease relapse. (Fig. 1)

Participants who completed FU1, completed $89 \%$ of weekly coaching phone calls and reported a median of 2 exercise sessions per week. All participants who completed FU1 measurements reported during interviews they would recommend the intervention to a friend or HCT survivor. 


\section{Body Composition}

Paired baseline and FU1 and baseline and FU2 DXA body composition data were available on 9 participants. Baseline body composition data were unable to be extracted from DXA scans on 2 participants. Of the participants with evaluable data at FU1, all either maintained $(n=3)$ or gained $(n=6)$ LBM at FU1 compared with baseline (Table 3). Lean body mass increased for all 8 participants with paired baseline and FU2 body composition data. The mean increase in LBM at FU1 was $1.1 \mathrm{~kg}(95 \%$ CI: $0.4,1.9)$ or $2 \%(95 \%$ CI: 1,4$)$. At FU2 the mean increase in LBM was $2.1 \mathrm{~kg}(95 \%$ CI: $0.5,3.8)$ or $5 \%$ (95\% CI: 1,8$)$. Percent fat mass at FU1 decreased for 3 participants, remained the same for 1 participant and increased for 5 participants. The mean increase from baseline in PFM at FU1 was $1 \%(95 \%$ CI -2, 5) and $1 \%$ (95\% CI -3, 5) at FU2. Of note, all participants who had an increase in PFM at FU1 were on oral prednisone for GVHD at some point during the intervention while all participants who decreased PFM or remained the same were not.

\section{Muscular Strength}

Of the participants who completed FU1, all increased their 6-minute walk distance with a $14 \%$ (95\% CI: 9, 20) mean increase from baseline (Table 4). 10 participants increased their 30-second chair stand score with the other participant obtaining the same score resulting in a mean $36 \%$ increase (95\% CI: 16, 57) from baseline. Dominant grip strength increased in 9 participants and non-dominant grip strength increased in 8 participants corresponding to a $14 \%$ (95\% CI: 5, 23) and 19\% (95\% CI 4, 33) mean increase respectively. One repetition maximum chest press increased in 10 participants and leg press one repetition max increased in 9 participants corresponding to a 30\% (95\% CI: 14, 47) and 26\% (95\% CI: 11, 41) mean increase respectively. (Fig. 2)

Of the participants who completed FU2, compared with baseline, all participants improved their 30-second chair stand score with a mean increase of $51 \%(95 \%$ CI: 12,89$)$ and 6 -minute walk test with a mean increase of $24 \%$ (95\% CI: 5, 42). Additionally, all participants increased their one repetition maximum chest press and leg press with a mean increase of $38 \%$ (95\% CI: 10,67) and 32\% (95\% CI: 4, 61) respectively. Dominant and non-dominant grip strength increased by a mean of $9 \%(95 \% \mathrm{CI}:-12,30)$ and $18 \%(95 \% \mathrm{CI}:-6,43)$.

\section{DISCUSSION}

We report the results of a pilot study, which demonstrates the feasibility and acceptability of a home-based RT intervention for AYA HCT survivors in the early post-HCT period. The feasibility goals of $>60 \%$ enrollment of eligible HCT survivors and completion of $>80 \%$ weekly phone coaching calls were achieved. Completion rates of the 12-week intervention among enrolled participants were lower than the anticipated $80 \%$ rate due to the fact that 4 participants dropped out of the study due to medical issues/relapse or SARS-CoV-2 pandemic reasons and 2 due to lack of evening or weekend hours at the FHCRC Exercise Research Center for initial teaching sessions. Promisingly, all participants increased or at least maintained lean body mass following the intervention (FU1) and at 1-year post-HCT (FU2). Furthermore, an overwhelming majority of patients also demonstrated improvements in functional strength assessments. Previous studies have demonstrated that following allo-HCT, total lean body mass significantly decreases corresponding to increased incidence of sarcopenia. ${ }^{25-27}$ Therefore, the resistance training intervention we describe in this study serves as a promising intervention to increase lean body mass and prevent sarcopenia.

In addition to the improvements in LBM, we also saw corresponding improvements in strength assessments of major muscle groups from baseline at both follow-up timepoints. These results demonstrate not only improvement in body composition but also function. However, without a control group, it is difficult to attribute improvements to the RT intervention versus natural improvements that may occur with the passage of time and growth after HCT. Despite seeing improvements in LBM, these improvements were not universally seen for changes in PFM. All of the participants who had an increase in PFM at FU1 ( $n=5)$ were on steroids for GVHD at some point during the intervention period. Therefore, the increase in PFM following the intervention could largely be due to the use of steroids for treatment of graft versus host disease. Future interventions could combine both resistance and aerobic exercise to target both improvements in LBM and percent fat mass. 
The intervention described above is unique in the fact that it utilizes both remote and in-person coaching, outpatient based, and is designed for the early post-HCT period. Previous literature to date has focused on older and young populations, as well as predominantly hospital-based interventions. A home-based resistance training intervention is particularly of value in this immunocompromised population which has been highlighted by the SARS-CoV-2 pandemic. Furthermore, as we also learned in our study, AYA survivors can find it hard to return to regional cancer centers for work, family and educational reasons, making a homebased intervention desirable in this population. We have learned that having weekend and evening hours available for teaching sessions and follow-up assessments is particularly important in the AYA population.

There are a number of limitations to our study, outside of the small number of participants, little racial and ethnic diversity, single transplant center, and the lack of a control group. Data regarding pre-HCT body composition were unavailable and not obtained as part of this study. Furthermore, we were unable to extract baseline body composition data from the DXA scans for 2 participants as only limited DXA scans for bone density were performed. Additionally, we did not collect data on nutrition and protein intake which can also have an impact on lean body mass gain.

This study provides preliminary evidence supporting a randomized controlled trial of a home-based resistance training exercise program in AYA survivors that is both feasible and acceptable. Future randomized trials are needed to confirm and validate these early findings. Given the prevalence of sarcopenia and metabolic syndrome observed in HCT survivors, exercise interventions that target skeletal muscle loss and cardiometabolic risk factors are needed to address the chronic metabolic complications in HCT survivors.

\section{CONFLICT OF INTEREST STATEMENT:}

The authors declare that there is no conflict of interest.

\section{ACKNOWLEDGMENTS:}

This investigation was supported by the National Institutes of Health under Ruth L. Kirschstein National Research Service Award T32CA00935, Cancer Center Support Grant (P30 CA015704) the National Cancer Institute Transdisciplinary Research on Energetics and Cancer (TREC) Training Workshop (R25CA203650, PI: Melinda Irwin), Conquer Cancer Foundation and the Seattle Children's Research Institute Clinical Research Scholars Program.

\section{REFERENCES}

1. Miller KD, Nogueira L, Mariotto AB, et al. Cancer treatment and survivorship statistics, 2019. CA Cancer J Clin . 2019;69(5):363-385. doi:10.3322/caac.21565

2. Oeffinger KC, Mertens AC, Sklar CA, et al. Chronic health conditions in adult survivors of childhood cancer. N Engl J Med . 2006;355(15):1572-1582. doi:10.1056/NEJMsa060185

3. Oeffinger KC, Buchanan GR, Eshelman DA, et al. Cardiovascular risk factors in young adult survivors of childhood acute lymphoblastic leukemia. J Pediatr Hematol Oncol . 2001;23(7):424-430.

4. Oeffinger KC, Adams-Huet B, Victor RG, et al. Insulin Resistance and Risk Factors for Cardiovascular Disease in Young Adult Survivors of Childhood Acute Lymphoblastic Leukemia. J Clin Oncol . 2009;27(22):3698. doi:10.1200/JCO.2008.19.7251

5. Oeffinger KC, Mertens AC, Sklar CA, et al. Obesity in Adult Survivors of Childhood Acute Lymphoblastic Leukemia: A Report from the Childhood Cancer Survivor Study. J Clin Oncol . 2003;21(7):1359-1365. doi:10.1200/JCO.2003.06.131

6. Majhail NS, Brazauskas R, Hassebroek A, et al. Outcomes of allogeneic hematopoietic cell transplantation for adolescent and young adults compared with children and older adults with acute myeloid leukemia.Biol Blood Marrow Transplant J Am Soc Blood Marrow Transplant . 2012;18(6):861-873. doi:10.1016/j.bbmt.2011.10.031 
7. Ketterl TG, Chow EJ, Leisenring WM, et al. Adipokines, Inflammation, and Adiposity in Hematopoietic Cell Transplantation Survivors.Biol Blood Marrow Transplant J Am Soc Blood Marrow Transplant . 2018;24(3):622-626. doi:10.1016/j.bbmt.2017.11.024

8. Baker KS, Ness KK, Steinberger J, et al. Diabetes, hypertension, and cardiovascular events in survivors of hematopoietic cell transplantation: a report from the bone marrow transplantation survivor study. Blood . 2007;109(4):1765-1772. doi:10.1182/blood-2006-05-022335

9. Baker KS, Chow E, Steinberger J. Metabolic syndrome and cardiovascular risk in survivors after hematopoietic cell transplantation. Bone Marrow Transplant . 2012;47(5):619-625. doi:10.1038/bmt.2011.118

10. Reusch JEB. Current concepts in insulin resistance, type 2 diabetes mellitus, and the metabolic syndrome. Am J Cardiol . 2002;90(5, Supplement 1):19-26. doi:10.1016/S0002-9149(02)02555-9

11. Ketterl TG, Chow EJ, Leisenring WM, et al. Adipokine Concentrations and Adiposity in Hematopoietic Cell Transplant (HCT) Survivors.Biol Blood Marrow Transplant . 2017;23(3):S67-S68. doi:10.1016/j.bbmt.2017.01.021

12. Wei C, Thyagiarajan MS, Hunt LP, Shield JPH, Stevens MCG, Crowne EC. Reduced insulin sensitivity in childhood survivors of haematopoietic stem cell transplantation is associated with lipodystropic and sarcopenic phenotypes. Pediatr Blood Cancer . 2015;62(11):1992-1999. doi:10.1002/pbc.25601

13. Dieli-Conwright CM, Courneya KS, Demark-Wahnefried W, et al. Effects of Aerobic and Resistance Exercise on Metabolic Syndrome, Sarcopenic Obesity, and Circulating Biomarkers in Overweight or Obese Survivors of Breast Cancer: A Randomized Controlled Trial. J Clin Oncol Off J Am Soc Clin Oncol . 2018;36(9):875-883. doi:10.1200/JCO.2017.75.7526

14. ACSM's resource manual for Guidelines for exercise testing and prescription | ACQUIRE Repository (3.4.3). Accessed August 22, 2017. http://acquire.cqu.edu.au:8080/vital/access/manager/Repository/cqu:4883

15. Nunes PRP, Barcelos LC, Oliveira AA, et al. Effect of resistance training on muscular strength and indicators of abdominal adiposity, metabolic risk, and inflammation in postmenopausal women: controlled and randomized clinical trial of efficacy of training volume. Age Dordr Neth . 2016;38(2):40. doi:10.1007/s11357016-9901-6

16. Liao C-D, Tsauo J-Y, Lin L-F, et al. Effects of elastic resistance exercise on body composition and physical capacity in older women with sarcopenic obesity: A CONSORT-compliant prospective randomized controlled trial. Medicine (Baltimore) . 2017;96(23). doi:10.1097/MD.0000000000007115

17. Artero EG, Lee D, Lavie CJ, et al. Effects of Muscular Strength on Cardiovascular Risk Factors and Prognosis. J Cardiopulm Rehabil Prev . 2012;32(6):351. doi:10.1097/HCR.0b013e3182642688

18. Vargas S, Petro JL, Romance R, et al. Comparison of changes in lean body mass with a strengthversus muscle endurance-based resistance training program. Eur J Appl Physiol . 2019;119(4):933-940. doi:10.1007/s00421-019-04082-0

19. Huang SW, Ku JW, Lin LF, Liao CD, Chou LC, Liou TH. Body composition influenced by progressive elastic band resistance exercise of sarcopenic obesity elderly women: a pilot randomized controlled trial. Eur J Phys Rehabil Med . Published online January 12, 2017. doi:10.23736/S1973-9087.17.04443-4

20. Benson AC, Torode ME, Singh MAF. Muscular strength and cardiorespiratory fitness is associated with higher insulin sensitivity in children and adolescents. Int J Pediatr Obes IJPO Off J Int Assoc Study Obes . 2006;1(4):222-231.

21. Munsie C, Ebert J, Joske D, Ackland T. The Benefit of Physical Activity in Adolescent and Young Adult Cancer Patients During and After Treatment: A Systematic Review. J Adolesc Young Adult Oncol . 
2019;8(5):512-524. doi:10.1089/jayao.2019.0013

22. Chamorro-Viña C, Ruiz JR, Santana-Sosa E, et al. Exercise during hematopoietic stem cell transplant hospitalization in children.Med Sci Sports Exerc . 2010;42(6):1045-1053. doi:10.1249/MSS.0b013e3181c4dac1

23. Järvelä LS, Kemppainen J, Niinikoski H, et al. Effects of a home-based exercise program on metabolic risk factors and fitness in long-term survivors of childhood acute lymphoblastic leukemia.Pediatr Blood Cancer . 2012;59(1):155-160. doi:10.1002/pbc.24049

24. Irwin ML, American College of Sports Medicine, eds. ACSM's Guide to Exercise and Cancer Survivorship . Human Kinetics; 2012.

25. DeFilipp Z, Troschel FM, Qualls DA, et al. Evolution of Body Composition Following Autologous and Allogeneic Hematopoietic Cell Transplantation: Incidence of Sarcopenia and Association with Clinical Outcomes. Biol Blood Marrow Transplant J Am Soc Blood Marrow Transplant . 2018;24(8):1741-1747. doi:10.1016/j.bbmt.2018.02.016

26. Frisk P, Rössner SM, Norgren S, Arvidson J, Gustafsson J. Glucose metabolism and body composition in young adults treated with TBI during childhood. Bone Marrow Transplant . 2011;46(10):1303-1308. doi:10.1038/bmt.2010.307

27. Slater ME, Steinberger J, Ross JA, et al. Physical Activity, Fitness, and Cardiometabolic Risk Factors in Adult Survivors of Childhood Cancer with a History of Hematopoietic Cell Transplantation. Biol Blood Marrow Transplant J Am Soc Blood Marrow Transplant . 2015;21(7):1278-1283. doi:10.1016/j.bbmt.2015.04.007

LEGENDS FIGURE 1 Flow of participants through the study.

FIGURE 2 Percent change in muscular strength from baseline (BL) to follow-up 1 (FU1) and follow-up 2 (FU2). Red boxes indicate means and gray bars indicate $95 \%$ confidence intervals.

\section{Hosted file}

TABLE 1.pdf available at https://authorea.com/users/393344/articles/506979-feasibility-andacceptability-of-a-home-based-resistance-training-intervention-in-adolescent-and-youngadult-hematopoietic-cell-transplant-survivors

\section{Hosted file}

TABLE 2.pdf available at https://authorea.com/users/393344/articles/506979-feasibility-andacceptability-of-a-home-based-resistance-training-intervention-in-adolescent-and-youngadult-hematopoietic-cell-transplant-survivors

\section{Hosted file}

TABLE 3.pdf available at https://authorea.com/users/393344/articles/506979-feasibility-andacceptability-of-a-home-based-resistance-training-intervention-in-adolescent-and-youngadult-hematopoietic-cell-transplant-survivors

\section{Hosted file}

TABLE 4.pdf available at https://authorea.com/users/393344/articles/506979-feasibility-andacceptability-of-a-home-based-resistance-training-intervention-in-adolescent-and-youngadult-hematopoietic-cell-transplant-survivors 


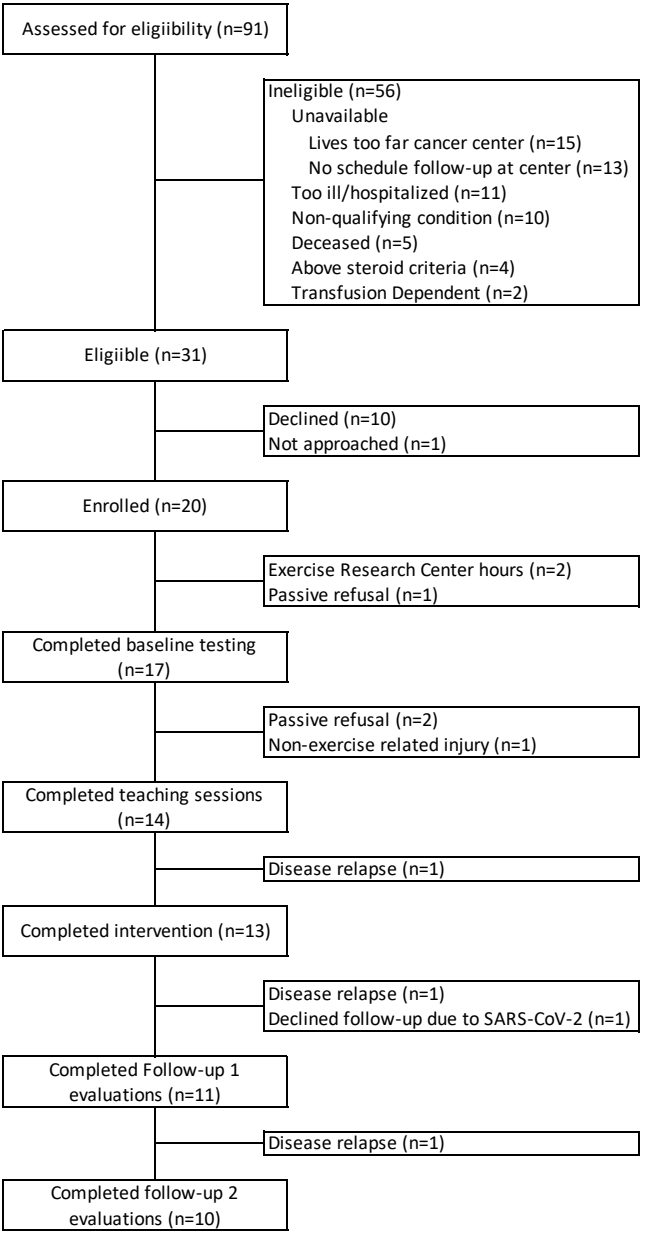




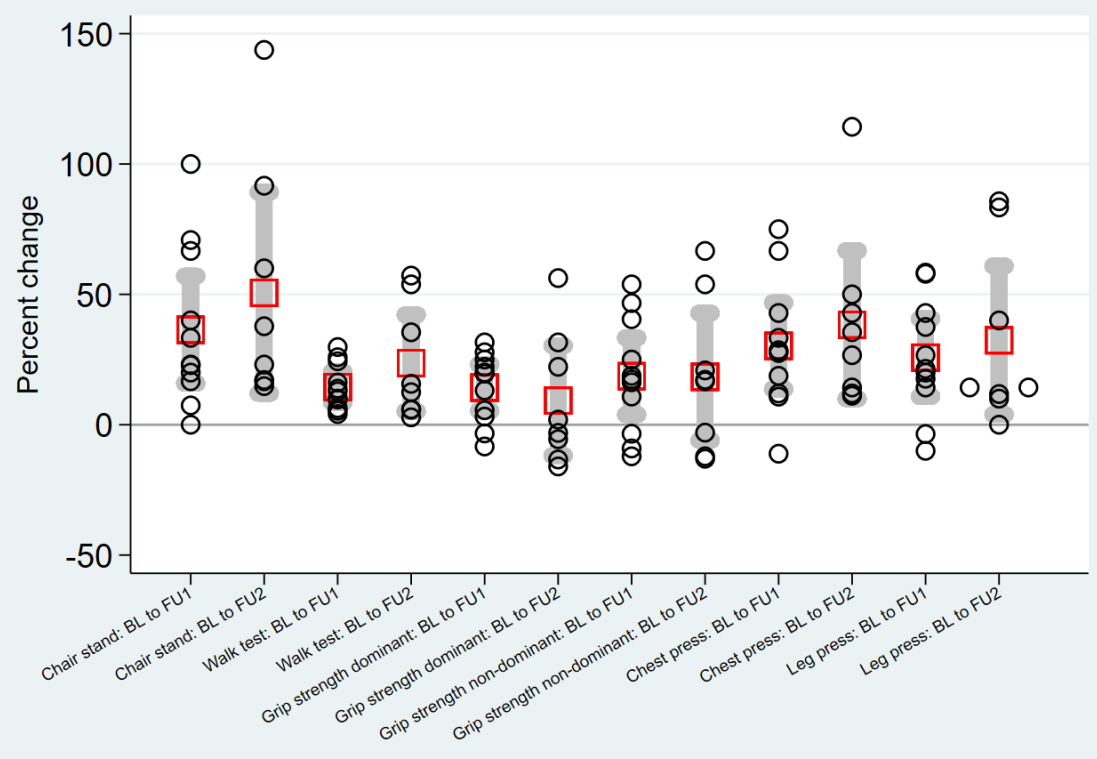

